
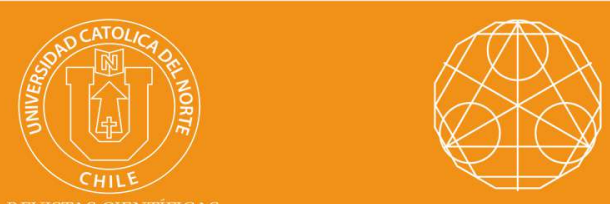

\title{
Computing the Schultz polynomials and indices for ladder related graphs
}

\begin{abstract}
Ali Ahmad* (iD orcid.org/0000-0003-3434-9908
*Jazan University, College of Computer Sci. \& Info. Technol., Jazan, Kingdom Saudi Arabia. ahmadsms@gmail.com
\end{abstract}

\section{Abstract:}

Distance is an important graph invariant that has wide applications in computing science and other fields of sciences. A topological index is a genuine number connected with compound constitution indicating for relationship of compound structure with different physical properties, synthetic reactivity or natural action. The Schultz and modified Schultz polynomials and their corresponding indices are used in synthetic graph theory as in light of vertex degrees. In this paper, the Schultz and modified

Keywords: Distance; Topological indices; Schultz indices; Schultz polynomial.

$\operatorname{MSC}(2010): 05 C 07,05 C 12,05 C 31$.

\section{Cite this article as (IEEE citation style):}

A. Ahmad, "Computing the Schultz polynomials and indices for ladder related graphs", Proyecciones (Antofagasta, On line), vol. 38, no. 5, pp. 1081-1092, Dec. 2019, doi: 10.22199/issn.0717-62792019-05-0070. [Accessed dd-mm-yyyy].

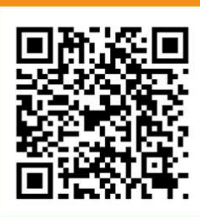

Article copyright: (C) 2019 Ali Ahmad. This is an open access article distributed under the terms of the Creative Commons Licence, which permits unrestricted use and distribution provided the original author and source are credited. 


\section{Introduction}

Let $G(V(G), E(G))$ be a simple connected undirected graph with vertex set $V(G)$ and edge set $E(G)$. Two vertices $u$ and $v$ in an undirected graph $G$ are called adjacent (or neighbors) in $G$ if $u$ and $v$ are endpoints of an edge $e$ of $G$. The degree of a vertex $u$ in an undirected simple graph, denoted as $d_{u}$ is the number of edges incident with it. The separation (distance) between vertices $u$ and $v ; d(u, v)$ is the quantity of edges in a most limited way associating them. The biggest separation between any two vertices of a graph $G$ is known as the diameter of $G$, denoted as $d(G)$. largest distance between any two vertices of a graph $G$ is called the diameter of $G$, denoted as $d(G)$.

While working on structural determination of the paraffin boiling points, Wiener [22] characterized a descriptor that is known as Wiener index. A great deal of topological indices have been presented, Wiener index is one of the topological indices that connect with a portion of the physico-chemical properties of the compound [1, 12]. Harry Shultz [19] presented another distance based topological index known as Shultz index with some of the physicochemical properties of the compound [1, 12]. Harry Shultz [19] introduced another distance based topological index known as Shultz index

$$
S c(G)=\sum_{\{u, v\} \subseteq V(G)}\left(d_{u}+d_{v}\right) d(u, v)
$$

In [16] showed the close relation between the Schultz index and the Wiener index. The modified Schultz index defined by Klavzar and Gutman [15] as:

$$
S c^{*}(G)=\sum_{\{u, v\} \subseteq V(G)}\left(d_{u} d_{v}\right) d(u, v)
$$

The modified Schultz index is closely related to the Wiener index as shown in [11]. $S c^{*}(G)=4 W(G)-n(2 n-1)$. Hosoya [14] acquainted a distancebased polynomial to generate distance distributions for graphs, called the Wiener polynomial

$$
H(G, x)=\sum_{\{u, v\} \subseteq V(G)} x^{d(u, v)}
$$

The first derivative of $H(G, x)$ at $x=1$ is equal to Wiener index of $G$. Gutman [11] introduced new polynomials called the Schultz polynomial and the modified Schultz polynomial as

$$
S c(G, x)=\sum_{\{u, v\} \subseteq V(G)}\left(d_{u}+d_{v}\right) x^{d(u, v)}
$$




$$
S c^{*}(G, x)=\sum_{\{u, v\} \subseteq V(G)}\left(d_{u} d_{v}\right) x^{d(u, v)}
$$

Such that their derivative at $x=1$ are equal to the Schultz and modified Schultz indices. He likewise acquired a few association between these polynomials and Wiener polynomial of trees. For further details we refer $[2,3,4,5,6,7,8,9,10,13,20]$.

The Schultz and modified Schultz polynomials and their corresponding indices were set up in engineered outline speculation in light of vertex degrees. In this paper, the Schultz and modified Schultz polynomials and their corresponding indices for Mongolian tent graph, diamond graph and double fan are computed.

\section{Results and Discussion}

The ladder graph, denoted by $L_{n}$, is the graph with vertex set

$$
V\left(L_{n}\right)=\left\{u_{i}, v_{i}: 1 \leq i \leq n\right\}
$$

and edge set

$$
E\left(L_{n}\right)=\left\{u_{i} u_{i+1}, v_{i} v_{i+1}: 1 \leq i \leq n-1\right\} \cup\left\{u_{i} v_{i}: 1 \leq i \leq n\right\} .
$$

$L_{n}$ is isomorphic to the grid $P_{2} \times P_{n}$.

Mongolian tent, denoted by $M t_{n}$, is the graph obtained from the ladder graph $L_{n}$ by adding a new vertex $z$ and joining each vertex $v_{i}, 1 \leq i \leq n$ with $z$. This graph is shown in Figure 1.

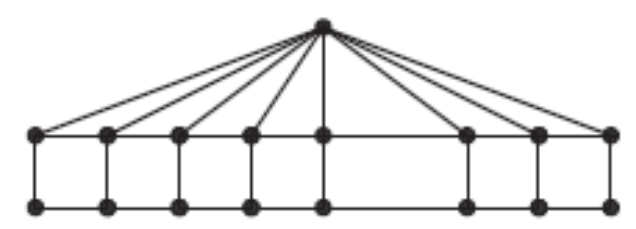

Figure 1: Mongolian tent graph $M t_{n}$

In the next theorem, the Schultz and modified Schultz polynomials and their corresponding indices of Mongolian tent graph are studied.

Theorem 1. Let $M t_{n}$ be a Mongolian tent graph with order $n \geq 3$. Then, 
- The Schultz polynomial and index of $M t_{n}$ are:

$S c\left(M t_{n}, x\right)=\left(n^{2}+25 n-24\right) x+\left(5 n^{2}+35 n-60\right) x^{2}+(72 n-188) x^{3}+$ $\left(3 n^{2}-17 n+18\right) x^{4}$

$S c\left(M t_{n}\right)=23 n^{2}+243 n-636$.

- The Modified Schultz polynomial and index of $M t_{n}$ are:

$S c^{*}\left(M t_{n}, x\right)=\left(4 n^{2}+35 n-51\right) x+\left(11 n^{2}+47 n-115\right) x^{2}+(115 n-$ $321) x^{3}+\left(\frac{9 n^{2}-57 n+74}{2}\right) x^{4}$

$S c^{*}\left(M t_{n}\right)=44 n^{2}+360 n-996$.

Proof. Consider the graph of Mongolian tent $M t_{n}$ with $n \geq 3$. The order of $M t_{n}$ is equal to $2 n+1$, in which 2 vertices of $M t_{n}$ have degree 2, $n$ vertices of $M t_{n}$ have degree $3, n-2$ vertices of $M t_{n}$ have degree 4 and only one vertex has degree $n$.

Thus, we divide the vertex set $V\left(M t_{n}\right)$ in four partitions:

$$
\begin{aligned}
& V_{2}=\left\{v \in V\left(M t_{n}\right): d_{v}=2\right\} \\
& V_{3}=\left\{v \in V\left(M t_{n}\right): d_{v}=3\right\} \\
& V_{4}=\left\{v \in V\left(M t_{n}\right): d_{v}=4\right\} \\
& V_{n}=\left\{v \in V\left(M t_{n}\right): d_{v}=n\right\}
\end{aligned}
$$

From Figure 1, the size of these four subsets are $\left|V_{2}\right|=2,\left|V_{3}\right|=3,\left|V_{4}\right|=$ $n-2$ and $\left|V_{n}\right|=1$. By using the hand shaking lemma the size of Mongolian tent graph $M t_{n}$ is equal to

$$
\left|E\left(M t_{n}\right)\right|=\frac{1}{2}\left[2 \times\left|V_{2}\right|+3 \times\left|V_{3}\right|+4 \times\left|V_{4}\right|+n \times\left|V_{n}\right|\right]=\frac{4+3 n+4 n-8+n}{2}=
$$
$4 n-2$.

From Figure 1, it is easy to see that for every vertices $u, v \in V\left(M t_{n}\right), \exists d(u, v) \in$ $\{1,2,3,4\}$.

Now, from the structure of the Mongolain tent graph $M t_{n}$, we compute all terms of the Schultz polynomial, modified Schultz polynomial of $M t_{n}$, based on the number of $d(u, v) \forall u, v \in V\left(M t_{n}\right)$.

Here, consider $d(u, v)=1\left(\forall u, v \in V\left(M t_{n}\right)\right)$, so from edge set $E\left(M t_{n}\right)$, we can see that there are 4 paths with length one or 4 edges $u v \in E\left(M t_{n}\right)$ for vertex $u \in V_{2} \subset V\left(M t_{n}\right)$ and a vertex $v \in V_{3} \subset V\left(M t_{n}\right)$ such that $d_{u}+d_{v}=5$ and $d_{u} \times d_{v}=6$. For a vertex $u \in V_{3} \subset V\left(M t_{n}\right)$, there are $n-3$ paths with length one until to a vertex $v \in V_{3}$ such that $d_{u}+d_{v}=3+3=6$ and $d_{u} \times d_{v}=3 \times 3=9$; there are $n$ 1-edges paths between the vertices $u \in V_{3} \subset V\left(M t_{n}\right)$ and $v \in V_{4} \subset V\left(M t_{n}\right)$ such that $d_{u}+d_{v}=3+4=7$, $d_{u} \times d_{v}=3 \times 4=12$, there are 2 1-edges paths between the vertices $u \in V_{3} \subset V\left(M t_{n}\right)$ and $v \in V_{n} \subset V\left(M t_{n}\right)$ such that $d_{u}+d_{v}=3+n=n+3$, $d_{u} \times d_{v}=3 \times n=3 n$; there are $n-31$-edges paths between the vertices 
$u, v \in V_{4} \subset V\left(M t_{n}\right)$ such that $d_{u}+d_{v}=4+4=8, d_{u} \times d_{v}=4 \times 4=16$; there are $n-2$ 1-edges paths between the vertices $u \in V_{4} \subset V\left(M t_{n}\right)$ and $v \in V_{n} \subset V\left(M t_{n}\right)$ such that $d_{u}+d_{v}=4+n=n+4, d_{u} \times d_{v}=4 \times n=4 n$. Therefore the first term of Schultz and modified Schultz polynomial of $M t_{n}$ will be $5(4) x+6(n-3) x+7(n) x+(n+3) 2 x+8(n-3) x+(n+4)(n-2) x=$ $\left(n^{2}+25 n-24\right) x$ and $6(4) x+9(n-3) x+12(n) x+(3 n) 2 x+16(n-3) x+$ $(4 n)(n-2) x=\left(4 n^{2}+35 n-51\right) x$, respectively.

In case $d(u, v)=2, u, v \in V\left(M t_{n}\right)$ : there are 2 2-edges paths between the vertices $u \in V_{2}$ and $v \in V_{3} \subset V\left(M t_{n}\right)$ such that $d_{u}+d_{v}=5, d_{u} \times d_{v}=$ $2 \times 3=6$; there are 4 2-edges paths between the vertices $u \in V_{2} \subset V\left(M t_{n}\right)$ and $v \in V_{4} \subset V\left(M t_{n}\right)$ such that $d_{u}+d_{v}=2+4=6, d_{u} \times d_{v}=2 \times 4=8$; there are 2 2-edges paths between the vertices $u \in V_{2} \subset V\left(M t_{n}\right)$ and $v \in V_{n} \subset V\left(M t_{n}\right)$ such that $d_{u}+d_{v}=2+n, d_{u} \times d_{v}=2 \times n=2 n$, there are $n+1$ 2-edges paths between the vertices $u, v \in V_{3} \subset V\left(M t_{n}\right)$ such that $d_{u}+d_{v}=3+3=6, d_{u} \times d_{v}=3 \times 3=9$; there are $6 n-14$ 2-edges paths between the vertices $u \in V_{3} \subset V\left(M t_{n}\right)$ and $v \in V_{4} \subset V\left(M t_{n}\right)$ such that $d_{u}+d_{v}=3+4=7, d_{u} \times d_{v}=3 \times 4=12$; there are $n-22$ edges paths between the vertices $u \in V_{3} \subset V\left(M t_{n}\right)$ and $v \in V_{n} \subset V\left(M t_{n}\right)$ such that $d_{u}+d_{v}=3+n=n+3, d_{u} \times d_{v}=3 \times n=3 n$. Finally, there are $n-4+\frac{(n-2)(n-4)}{2}$ 2-edges paths between all vertices $u, v \in V_{4} \subset$ $V\left(M t_{n}\right)$ such that $d_{u}+d_{v}=4+4=8, d_{u} \times d_{v}=4 \times 4=16$. Then the second term of Schultz and modified Schultz polynomial of $M t_{n}$ is equal to $\left[10+24+2 n+4+6 n+6+7(6 n-14)+(n+3)(n-2)+8\left(n-4+\frac{(n-2)(n-4)}{2}\right)\right] x^{2}=$ $\left(5 n^{2}+35 n-60\right) x^{2}$ and $[12+32+4 n+9 n+9+12(6 n-14)+3 n(n-2)+$ $\left.16\left(n-4+\frac{(n-2)(n-4)}{2}\right)\right] x^{2}=\left(11 n^{2}+47 n-115\right) x^{2}$, respectively.

In case $d(u, v)=3, u, v \in V\left(M t_{n}\right)$ : there are 23 -edges paths between the vertices $u \in V_{2}$ and $v \in V_{3} \subset V\left(M t_{n}\right)$ such that $d_{u}+d_{v}=5, d_{u} \times d_{v}=$ $2 \times 3=6$; there are $2 n 3$-edges paths between the vertices $u \in V_{2} \subset V\left(M t_{n}\right)$ and $v \in V_{4} \subset V\left(M t_{n}\right)$ such that $d_{u}+d_{v}=2+4=6, d_{u} \times d_{v}=2 \times 4=8$; there are $3 n-53$-edges paths between the vertices $u, v \in V_{3} \subset V\left(M t_{n}\right)$ such that $d_{u}+d_{v}=3+3=6, d_{u} \times d_{v}=3 \times 3=9$. Finally, there are $6 n-243$-edges paths between the vertices $u \in V_{3} \subset V\left(M t_{n}\right)$ and $u \in V_{4} \subset V\left(M t_{n}\right)$ such that $d_{u}+d_{v}=3+4=7, d_{u} \times d_{v}=3 \times 4=12$. Then the third term of Schultz and modified Schultz polynomial of $M t_{n}$ is equal to $[10+12 n+6(3 n-5)+7(6 n-24)] x^{3}=(72 n-188) x^{3}$ and $[12+16 n+9(3 n-5)+12(6 n-24)] x^{3}=(115 n-321) x^{3}$, respectively.

In case $d(u, v)=4, u, v \in V\left(M t_{n}\right)$ : there are 14 -edges paths between all vertices $u, v \in V_{2} \subset V\left(M t_{n}\right)$ such that $d_{u}+d_{v}=2+2=4, d_{u} \times d_{v}=$ 
$2 \times 2=4$; there are $2 n-8$ 4-edges paths between the vertices $u \in V_{2} \subset$ $V\left(M t_{n}\right)$ and $v \in V_{3} \subset V\left(M t_{n}\right)$ such that $d_{u}+d_{v}=2+3=5, d_{u} \times d_{v}=$ $2 \times 3=6$. Finally, there are $\frac{n^{2}-9 n+18}{2} 4$-edges paths between all vertices $u, v \in V_{3} \subset V\left(M t_{n}\right)$ such that $d_{u}+d_{v}=3+3=6, d_{u} \times d_{v}=3 \times 3=9$. Then the fourth term of Schultz and modified Schultz polynomial of $M t_{n}$ is equal to $\left[4+5(2 n-8)+6\left(\frac{n^{2}-9 n+18}{2}\right)\right] x^{4}=\left(3 n^{2}-17 n+18\right) x^{4}$ and $\left[4+6(2 n-8)+9\left(\frac{n^{2}-9 n+18}{2}\right)\right] x^{4}=\left(\frac{9 n^{2}-57 n+74}{2}\right) x^{4}$, respectively.

Hence, Schultz and modified Schultz polynomials of $M t_{n}$ are:

$$
\begin{aligned}
& S c\left(M t_{n}, x\right)=\sum_{u, v \in V\left(M t_{n}\right)}\left(d_{u}+d_{v}\right) x^{d(u, v)} \\
& =\left(n^{2}+25 n-24\right) x+\left(5 n^{2}+35 n-60\right) x^{2}+(72 n-188) x^{3}+\left(3 n^{2}-17 n+18\right) x^{4} \\
& S c^{*}\left(M t_{n}, x\right)=\sum_{u, v \in V\left(M t_{n}\right)}\left(d_{u} \times d_{v}\right) x^{d(u, v)}
\end{aligned}
$$

By definitions of the Schultz and modified Schultz indices, we have

$$
S c\left(M t_{n}\right)=\left.\frac{\partial S c\left(M t_{n}, x\right)}{\partial x}\right|_{x=1}
$$

$=\frac{\partial}{\partial x}\left(\left(n^{2}+25 n-24\right) x+\left(5 n^{2}+35 n-60\right) x^{2}+(72 n-188) x^{3}+\left(3 n^{2}-17 n+\right.\right.$ 18) $\left.x^{4}\right)\left.\right|_{x=1}$

$=23 n^{2}+243 n-636$.

$S c^{*}\left(M t_{n}\right)=\left.\frac{\partial S c^{*}\left(M t_{n}, x\right)}{\partial x}\right|_{x=1}$

$=\frac{\partial}{\partial x}\left(\left(4 n^{2}+35 n-51\right) x+\left(11 n^{2}+47 n-115\right) x^{2}+(115 n-321) x^{3}+\right.$ $\left.\left(\frac{9 n^{2}-57 n+74}{2}\right) x^{4}\right)\left.\right|_{x=1}$

$=44 n^{2}+360 n-996$. Which completes the proof.

$\square$ Diamond graph, denoted by $D_{n}$, is the graph obtained from the Mongolian tent graph $M t_{n}$ by adding a new vertex $z_{1}$ and joining each vertex $x_{i}, 1 \leq i \leq n$ with $z_{1}$. This graph is shown in Figure 2. The Schultz and modified Schultz polynomials and their corresponding indices of Diamond are determined in the following theorem. 


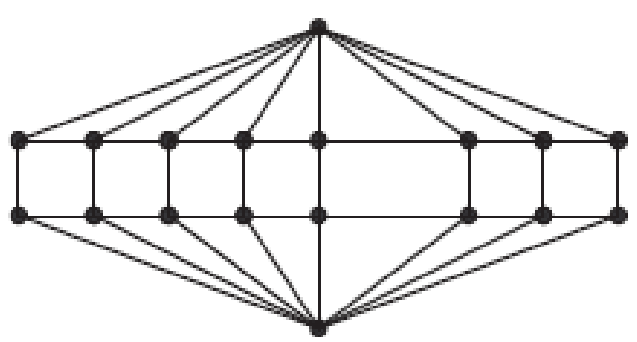

Figure 2: Diamond graph $D_{n}$

Theorem 2. Let $D_{n}$ be a Diamond graph with order $n \geq 3$. Then,

- The Schultz polynomial and index of $D_{n}$ are:

$S c\left(D_{n}, x\right)=\left(2 n^{2}+32 n-28\right) x+\left(10 n^{2}+28 n-42\right) x^{2}+\left(18 n^{2}-24 n-\right.$ 24) $x^{3}$

$\operatorname{Sc}\left(D_{n}\right)=76 n^{2}+16 n-184$.

- The Modified Schultz polynomial and index of $D_{n}$ are:

$S c^{*}\left(D_{n}, x\right)=\left(8 n^{2}+44 n-62\right) x+\left(24 n^{2}+28 n-86\right) x^{2}+\left(n^{3}+32 n^{2}-\right.$ $64 n-28) x^{3}$

$S c^{*}\left(D_{n}\right)=3 n^{3}+152 n^{2}-92 n-318$.

Proof. Consider the graph of Diamond $D_{n}$ with $n \geq 3$. The order of $D_{n}$ is equal to $2 n+2$, in which 4 vertices of $D_{n}$ have degree $3,2 n-4$ vertices of $D_{n}$ have degree 4 and two vertices have degree $n$.

Thus, we divide the vertex set $V\left(D_{n}\right)$ in three partitions:

$V_{3}=\left\{v \in V\left(D_{n}\right): d_{v}=3\right\}$

$V_{4}=\left\{v \in V\left(D_{n}\right): d_{v}=4\right\}$

$V_{n}=\left\{v \in V\left(D_{n}\right): d_{v}=n\right\}$

From Figure 2, the size of these three subsets are $\left|V_{3}\right|=4,\left|V_{4}\right|=2 n-4$ and $\left|V_{n}\right|=2$. By using the hand shaking lemma the size of Diamond graph $D_{n}$ is equal to

$\left|E\left(D_{n}\right)\right|=\frac{1}{2}\left[3 \times\left|V_{3}\right|+4 \times\left|V_{4}\right|+n \times\left|V_{n}\right|\right]=\frac{12+8 n-8+2 n}{2}=5 n-2$.

From Figure 2, we can see that there are distance between vertices of graph $D_{n}$ are up to three and the diameter equal to 3 .

Now, from the structure of the Diamond graph $D_{n}$, we compute all terms of the Schultz polynomial, modified Schultz polynomial of $D_{n}$, based on the number of $d(u, v) \forall u, v \in V\left(D_{n}\right)$. 
Table 2.1: All cases of $d(u, v)$-edge-paths $d(u, v)=1,2,3$ of the Diamond graph $D_{n}$.

\begin{tabular}{lcccc}
\hline $\begin{array}{l}\text { distance } \\
d(u, v)=i\end{array}$ & $\begin{array}{c}\text { degrees of } \\
d_{u} \& d_{v}\end{array}$ & $\begin{array}{c}\text { Number of } \\
\text { i-edges paths }\end{array}$ & $\begin{array}{c}\text { Term of Schultz } \\
\text { polynomial }\end{array}$ & $\begin{array}{c}\text { Term of Modified } \\
\text { Schultz polynomial }\end{array}$ \\
\hline 1 & $(3,3)$ & 2 & 12 & 18 \\
& $(3,4)$ & 4 & 28 & 48 \\
& $(3, n)$ & 4 & $4 n+12$ & $12 n$ \\
& $(4,4)$ & $3 n-8$ & $24 n-64$ & $48 n-128$ \\
& $(4, n)$ & $2 n-4$ & $2 n^{2}+4 n-16$ & $8 n^{2}-16 n$ \\
\hline 2 & $(3,3)$ & 2 & 12 & 18 \\
& $(3,4)$ & $4 n+2$ & $28 n+14$ & $48 n+24$ \\
& $(3, n)$ & 4 & $4 n+12$ & $12 n$ \\
& $(4,4)$ & $n^{2}-n-8$ & $8 n^{2}-8 n-64$ & $16 n^{2}-16 n-128$ \\
& $(4, n)$ & $2 n-4$ & $2 n^{2}+4 n-16$ & $8 n^{2}-16 n$ \\
\hline 3 & $(3,3)$ & 4 & 24 & 36 \\
& $(3,4)$ & $8 n-16$ & $56 n-112$ & $96 n-192$ \\
& $(4,4)$ & $2 n^{2}-10 n+8$ & $16 n^{2}-80 n+64$ & $32 n^{2}-160 n+128$ \\
& $(n, n)$ & $n$ & $2 n^{2}$ & $n^{3}$ \\
\hline
\end{tabular}

By using the Table 1, we obtain the followings:

- coefficient first term of the Schultz polynomial: $2 n^{2}+32 n-28$

- coefficient second term of the Schultz polynomial: $10 n^{2}+28 n-42$

- coefficient third term of the Schultz polynomial: $18 n^{2}-24 n-24$

- coefficient first term of the modified Schultz polynomial: $8 n^{2}+44 n-62$

- coefficient second term of the modified Schultz polynomial: $24 n^{2}+$ $28 n-86$

- coefficient third term of the modified Schultz polynomial: $n^{3}+32 n^{2}-$ $64 n-28$

Hence, Schultz and modified Schultz polynomials of $D_{n}$ are:

$$
\begin{aligned}
& S c\left(D_{n}, x\right)=\sum_{u, v \in V\left(D_{n}\right)}\left(d_{u}+d_{v}\right) x^{d(u, v)} \\
& =\left(2 n^{2}+32 n-28\right) x+\left(10 n^{2}+28 n-42\right) x^{2}+\left(18 n^{2}-24 n-24\right) x^{3} \\
& S c^{*}\left(D_{n}, x\right)=\sum_{u, v \in V\left(D_{n}\right)}\left(d_{u} \times d_{v}\right) x^{d(u, v)}
\end{aligned}
$$


$=\left(8 n^{2}+44 n-62\right) x+\left(24 n^{2}+28 n-86\right) x^{2}+\left(n^{3}+32 n^{2}-64 n-28\right) x^{3}$

By definitions of the Schultz and modified Schultz indices, we have $S c\left(D_{n}\right)=\left.\frac{\partial S c\left(D_{n}, x\right)}{\partial x}\right|_{x=1}$

$=\left.\frac{\partial}{\partial x}\left(\left(2 n^{2}+32 n-28\right) x+\left(10 n^{2}+28 n-42\right) x^{2}+\left(18 n^{2}-24 n-24\right) x^{3}\right)\right|_{x=1}$

$=76 n^{2}+16 n-184$.

$S c^{*}\left(D_{n}\right)=\left.\frac{\partial S c^{*}\left(D_{n}, x\right)}{\partial x}\right|_{x=1}$

$=\left.\frac{\partial}{\partial x}\left(\left(8 n^{2}+44 n-62\right) x+\left(24 n^{2}+28 n-86\right) x^{2}+\left(n^{3}+32 n^{2}-64 n-28\right) x^{3}\right)\right|_{x=1}$

$=3 n^{3}+152 n^{2}-92 n-318$. Which completes the proof.

Fan graph, denoted by $f_{n}$, is the graph obtained from the path with $n$ vertices $P_{n}$, where $V\left(P_{n}\right)=\left\{v_{1}, v_{2}, \ldots, v_{n}\right\}$ and $E\left(P_{n}\right)=\left\{v_{i} v_{i+1}: 1 \leq i \leq\right.$ $n-1\}$ by adding a new vertex $z$ and joining each $v_{i}, 1 \leq i \leq n$ with $z$.

Double fan graph, denoted by $d f_{n}$, is the graph obtained from the fan $f_{n}$ by adding a new vertex $z_{1}$ joining each $v_{i}, 1 \leq i \leq n$ with $z_{1}$.

Theorem 3. Let $d f_{n}$ be a double fan graph with order $n \geq 5$. Then,

- The Schultz polynomial and index of $d f_{n}$ are:

$S c\left(d f_{n}, x\right)=\left(2 n^{2}+16 n-14\right) x+\left(18 n^{2}-76 n-218\right) x^{2}$

$S c\left(d f_{n}\right)=38 n^{2}-136 n-450$.

- The Modified Schultz polynomial and index of $d f_{n}$ are:

$S c^{*}\left(d f_{n}, x\right)=\left(8 n^{2}+12 n-24\right) x+\left(n^{3}+32 n^{2}-160 n-422\right) x^{2}$

$S c^{*}\left(d f_{n}\right)=2 n^{3}+72 n^{2}-308 n-868$.

Proof. Consider the graph of double fan $d f_{n}$ with $n \geq 5$. The order of $d f_{n}$ is equal to $n+2$, in which 2 vertices of $d f_{n}$ have degree $3, n-2$ vertices of $d f_{n}$ have degree 4 and two vertices have degree $n$.

Thus, we divide the vertex set $V\left(d f_{n}\right)$ in three partitions:

$V_{3}=\left\{v \in V\left(d f_{n}\right): d_{v}=3\right\}$

$V_{4}=\left\{v \in V\left(d f_{n}\right): d_{v}=4\right\}$

$V_{n}=\left\{v \in V\left(d f_{n}\right): d_{v}=n\right\}$

The size of these three subsets are $\left|V_{3}\right|=2,\left|V_{4}\right|=n-2$ and $\left|V_{n}\right|=2$.

By using the hand shaking lemma the size of double fan $d f_{n}$ is equal to

$\left|E\left(d f_{n}\right)\right|=\frac{1}{2}\left[3 \times\left|V_{3}\right|+4 \times\left|V_{4}\right|+n \times\left|V_{n}\right|\right]=\frac{6+4 n-8+2 n}{2}=3 n-2$.

The diameter of double fan $d f_{n}$ equal to 2 .

Now, from the structure of the double fan $d f_{n}$, we compute all terms of the Schultz polynomial, modified Schultz polynomial of $d f_{n}$, based on the number of $d(u, v) \forall u, v \in V\left(d f_{n}\right)$. 
Table 2.2: All cases of $d(u, v)$-edge-paths $d(u, v)=1,2$ of the double fan $d f_{n}$.

\begin{tabular}{lcccc}
\hline $\begin{array}{l}\text { distance } \\
d(u, v)=i\end{array}$ & $\begin{array}{c}\text { degrees of } \\
d_{u} \& d_{v}\end{array}$ & $\begin{array}{c}\text { Number of } \\
\text { i-edges paths }\end{array}$ & $\begin{array}{c}\text { Term of Schultz } \\
\text { polynomial }\end{array}$ & $\begin{array}{c}\text { Term of Modified } \\
\text { Schultz polynomial }\end{array}$ \\
\hline 1 & $(3,4)$ & 2 & 14 & 24 \\
& $(3, n)$ & 4 & $4 n+12$ & $12 n$ \\
& $(4,4)$ & $n-3$ & $8 n-24$ & $16 n-48$ \\
& $(4, n)$ & $2 n-4$ & $2 n^{2}+4 n-16$ & $8 n^{2}-16 n$ \\
\hline 2 & $(3,3)$ & 2 & 12 & 18 \\
& $(3,4)$ & $4 n-10$ & $28 n-70$ & $48 n-120$ \\
& $(4,4)$ & $2 n^{2}-13 n+20$ & $16 n^{2}-104 n-160$ & $32 n^{2}-208 n-320$ \\
& $(n, n)$ & $n$ & $2 n^{2}$ & $n^{3}$ \\
\hline
\end{tabular}

By using the Table 2, we obtain the followings:

- coefficient first term of the Schultz polynomial: $2 n^{2}+16 n-14$

- coefficient second term of the Schultz polynomial: $18 n^{2}-76 n-218$

- coefficient first term of the modified Schultz polynomial: $8 n^{2}+12 n-24$

- coefficient second term of the modified Schultz polynomial: $n^{3}+32 n^{2}-$ $160 n-422$

Hence, Schultz and modified Schultz polynomials of $d f_{n}$ are:

$S c\left(d f_{n}, x\right)=\sum_{u, v \in V\left(d f_{n}\right)}\left(d_{u}+d_{v}\right) x^{d(u, v)}$

$=\left(2 n^{2}+16 n-14\right) x+\left(18 n^{2}-76 n-218\right) x^{2}$

$S c^{*}\left(d f_{n}, x\right)=\sum_{u, v \in V\left(d f_{n}\right)}\left(d_{u} \times d_{v}\right) x^{d(u, v)}$

$=\left(8 n^{2}+12 n-24\right) x+\left(n^{3}+32 n^{2}-160 n-422\right) x^{2}$

By definitions of the Schultz and modified Schultz indices, we have

$S c\left(d f_{n}\right)=\left.\frac{\partial S c\left(d f_{n}, x\right)}{\partial x}\right|_{x=1}$

$=\left.\frac{\partial}{\partial x}\left(\left(2 n^{2}+16 n-14\right) x+\left(18 n^{2}-76 n-218\right) x^{2}\right)\right|_{x=1}$

$=38 n^{2}-136 n-450$.

$S c^{*}\left(d f_{n}\right)=\left.\frac{\partial S c^{*}\left(d f_{n}, x\right)}{\partial x}\right|_{x=1}$

$=\left.\frac{\partial}{\partial x}\left(\left(8 n^{2}+12 n-24\right) x+\left(n^{3}+32 n^{2}-160 n-422\right) x^{2}\right)\right|_{x=1}$

$=2 n^{3}+72 n^{2}-308 n-868$. Which completes the proof. 


\section{Closing Remarks}

In this paper, we proved Schultz and modified Schultz polynomials for Mongolian tent graph $\left(M t_{n}\right)$, Diamond graph $\left(D_{n}\right)$, double fan $\left(d f_{n}\right)$. In future, we are interested to plan some beginning designs/systems and after that review their topological indices which will be very helper to comprehend their hidden topology.

\section{References}

[1] A. Dobrynin, R. Entringer and I Gutman, "Wiener index of trees: theory and applications", Acta applicandae mathematicae, vol. 66, no. 3 pp. 211-249, May 2001, doi: 10.1023/A:1010767517079.

[2] M. Eliasi and B. Taeri, "Schultz polynomials of composite graphs", Applicable analysis and discrete mathematics, vol. 2, no. 2, pp. 285-296, Apr. 2008. [On line]. Available: https://bit.ly/34yFuvy

[3] M. Farahani and M. Vlad, "On the Schultz, modified Schultz and Hosoya polynomials and derived indices of capradesigned planar benzenoid", Studia universitatis Babeş-Bolyai, chemia, vol. 57, no. 4, pp. 55-63, 2012. [On line]. Available: https://bit.ly/2YWgSvK

[4] M. Farahani, "Hosoya, Schultz, modified Schultz polynomials and their topological indices of benzene molecules, first members of polycyclic aromatic hydrocarbons (PAHs)", International journal of theoretical chemistry, vol. 1, no. 2, pp. 9-16, Oct. 2013. [On line]. Available: https://bit.ly/2S4WwPC

[5] M. Farahani, "On the Schultz and modified Schultz polynomials of some harary graphs", International journal of applications of discrete mathematics, vol. 1, no. 1, pp. 1-8, Sep. 2013. [On line]. Available: https://bit.ly/2r4PgIl

[6] M. Farahani, "On the Schultz polynomial and Hosoya polynomial of circumcoronene series of benzenoid", Journal of applied mathematics \& informatics, vol. 31, no. 5-6, pp. 595-608, 2013, doi: 10.14317/jami.2013.595.

[7] M. Farahani, "Schultz indices and Schultz polynomials of Harary graph", Pacific journal of applied mathematics, vol. 6, no. 3, pp. 77-84, 2014.

[8] M. Farahani and W. Gao, "The Schultz index and Schultz polynomial of the Jahangir Graphs J5,m", Applied mathematics, vol. 6, pp. 2319-2325, Dic. 2015, doi: 10.4236/am.2015.614204.

[9] M. Farahani, M. Kanna and W. Gao, "The Schultz, modified Schultz indices and their polynomials of the Jahangir graphs Jn,m for integer numbers $\mathrm{n}=3, \mathrm{~m} \geq 3$,, Asian journal of applied sciences, vol. 3, no. 6, pp. 823827, Dec. 2015. [On line]. Available: https://bit.ly/2PxBF5w

[10] M. Farahani and M. Jamil, "The Schultz and modified Schultz polynomials of certain subdivision and line subdivision graphs", Journal of chemical and pharmaceutical research, vol. 8, no. 3, pp. 51-57, 2016. [On line]. Available: https://bit.ly/2M55j08 
[11] I. Gutman, "Selected properties of the Schultz molecular topological index", Journal of chemical information and modeling, vol. 34, no. 5, pp. 1087-1089, Sep. 1994, doi: 10.1021/ci00021a009.

[12] I. Gutman and O. Polansky, Mathematical concepts in organic chemistry, Berlin: Springer, 1986, doi: 10.1007/978-3-642-70982-1.

[13] F. Hassani, A. Iranmanesh and S. Mirzaie, "Schultz and modified Schultz polynomials of C100 Fullerene", MATCH communications in mathematical and in computer chemistry, vol. 69, no. 1 pp. 87-92, 2013. [On line]. Available: https://bit.ly/2PWgSrA

[14] H. Hosoya, "On some counting polynomials in chemistry", Discrete applied mathematics, vol. 19, no. 1, pp. 239-257, Mar. 1988, doi: 10.1016/0166-218X(88)90017-0.

[15] S. Klavžar and I. Gutman, "Wiener number of vertex-weighted graphs and a chemical application", Discrete applied mathematics, vol. 80, no. 1, pp. 73-81, Dec. 1997, doi: 10.1016/S0166-218X(97)00070-X.

[16] D. Klein, Z. Mihalić, D. Plavšić, N. Trinjastić, "Molecular topological index: a relation with the Wiener index", Journal of chemical information and modeling, vol. 32, no. 4, pp. 304-305, Jul. 1992, doi: 10.1021/ci00008a008.

[17] M. Nadeem, S. Zafar and Z. Zahid, "On certain topological indices of the line graph of subdivision graphs", Applied mathematics and computation, vol. 271, pp. 790-794, Nov. 2015, doi: 10.1016/j.amc.2015.09.061.

[18] M. Nadeem, S. Zafar and Z. Zahid,"On topological properties of the line graphs of subdivision graphs of certain nanostructures", Applied mathematics and computation, vol. 273, pp. 125-130, Jan. 2016, doi: 10.1016/j.amc.2015.10.010.

[19] H. Schultz, "Topological organic chemistry 1. Graph theory and topological indices of alkanes", Journal of chemical information and modeling, vol. 29, no. 3, pp. 227-228, Aug. 1989, doi: 10.1021/ci00063a012.

[20] M. Siddiqui, M. Imran and A. Ahmad, "On Zagreb indices, Zagreb polynomials of some nanostar dendrimers", Applied mathematics and computation, vol. 280, pp. 132-139, Apr. 2016, doi: 10.1016/j.amc.2016.01.041.

[21] G. Su and L. Xu, "Topological indices of the line graph of subdivision graphs and their Schur-bounds", Applied mathematics and computation, vol. 253, pp. 395-401, Feb. 2015, doi: 10.1016/j.amc.2014.10.053.

[22] H. Wiener, "Structural determination of the paraffin boiling points", Journal of the American chemical society, vol. 69, no. 1, pp. 17-20, Jan. 1947, doi: 10.1021/ja01193a005. 